\title{
Revisiting the Marshmallow Test: On the Interpretation of Replication Results
}

\author{
Armin Falk ${ }^{1}$, Fabian Kosse ${ }^{2 *}$, Pia Pinger ${ }^{3}$ \\ ${ }^{1}$ Institute on Behavior and Inequality (briq) \& Department of Economics, University of Bonn \\ ${ }^{2}$ Department of Economics, LMU Munich \& Institute on Behavior and Inequality (briq) \\ ${ }^{3}$ Department of Economics, University of Cologne \& Institute on Behavior and Inequality (briq) \\ * Corresponding author (fabian.kosse@econ.Imu.de)
}

September 2019

\begin{abstract}
In a recent paper, Watts, Duncan and Quan (2018; henceforth WDQ) replicate and extend the famous marshmallow studies. Their paper seemingly challenges the original evidence. We acknowledge WDQs contribution, but raise two concerns regarding WDQ's findings and their interpretation. First, differences in measures between the two studies preclude a direct comparison of results. Most importantly, Monte Carlo Simulations indicate that the correlations reported in WDQ are biased towards zero due to censoring in the length of feasible waiting times. Second, a reduction in correlation size and significance in the presence of covariates which are not entirely exogenous or predetermined is difficult to interpret. We conclude that WDQ's findings cannot serve to refute the evidence reported by Mischel and Shoda and suggest that assessing the importance of delay of gratification for later life outcomes ultimately requires more refined models of the skill accumulation process as well as targeted intervention studies.
\end{abstract}


In a recent paper, Watts, Duncan and Quan (2018; henceforth WDQ) replicate and extend the famous marshmallow studies (Mischel, Shoda, \& Rodriguez, 1989; Shoda, Mischel, \& Peake, 1990; henceforth SMP), which found suggestive evidence of a substantial correlation between children's delay of gratification and later life outcomes almost 20 years ago. WDQ make an important contribution. They repeat the analysis on a larger and more diverse sample of children, with data that allow them to control for a substantial set of variables. Their findings seemingly challenge the original evidence, as the bivariate correlation between waiting times at the age of 4 and adolescent achievement in their data "[...] was only half the size of those reported in the original studies and was reduced by two thirds in the presence of controls for family background, early cognitive ability, and the home environment."

The aim of this commentary is not to downplay WDQ's contribution, but to raise two concerns that we hope will put their somewhat strong conclusions into perspective. First, differences in measures between the two studies preclude a direct comparison of results. Most importantly, due to more pronounced censoring in the length of feasible waiting times, the bivariate correlations reported in WDQ are biased towards zero. Second, a reduction in correlation size and significance in models with covariates that are not entirely exogenous or predetermined is difficult to interpret. A reanalysis of the data with predetermined conditioning variables yields estimates which are closer to the ones reported in the original studies.

\section{Differences in measures and bivariate correlations}

WDQ describe their paper as a "conceptual replication", since the underlying population as well as the achievement tests and delay of gratification measures substantially differ from SMP. In many respects, this obstructs a direct comparison of results, as exemplified in the following. Most importantly, the delay of gratification measure is based on a different experimental protocol, as the children in WDQ had to wait only for 7 minutes as opposed to 15 minutes in SMP. This variation of the task produces a heavily compressed distribution in the ability to delay gratification measure (standard deviation $=3.08 \mathrm{~min}$ ) compared with the SMP sample (standard deviation $=6.23 \mathrm{~min}$ ). The reason is that WDQ's data contain large fractions of censored observations ( $55 \%$ of children waited until the interviewer returned) while in 
the "diagnostic condition" of the original marshmallow study censoring is very low (Mischel, Ebbesen, \& Zeiss, 1972). WDQ acknowledge and address the censoring problem, but argue that it "does not affect the conclusion" (p.11), since the delay of gratification effect was only driven by children who waited less than two minutes. However, this statement hinges on nonmonotonicities in regression models with a substantial set of control variables (for a discussion on the control variables see below). It does not hold with respect to the bivariate relationships among waiting times and achievement (WDQ, Table 4, cols (1), (4)) that was used to compare effect sizes across papers. Above and beyond, these nonmonotonicities are not per se informative about the extent of censoring bias in correlation coefficients. In Monte Carlo simulations, detailed below, we show that the bivariate correlations in WDQ are considerably downwardbiased.

\section{METHODS AND RESULTS I}

To provide some guidance for a more direct comparison of the bivariate correlations for achievement measures reported in SMP and WDQ, in Table 1, we use Monte Carlo simulations to illustrate the downward-biasing effect of censoring on correlation coefficients. We answer the following question: assuming true bivariate correlations of the size reported in SMP, how does censoring affect the estimated correlation coefficients? The corresponding confidence intervals reflect degrees of uncertainty for samples of the size reported in SMP and WDQ, respectively. The simulation results indicate that in the presence of $55 \%$ censoring, as in the full sample of WDQ, the correlations in SMP $\left(\rho_{\text {SMP }}(35)=0.42\right.$ for SAT Verbal, respectively, 0.57 for SAT Quantitative) would be much lower $\left(\rho_{\text {SMP }}^{\prime}(35)=0.349\right.$, respectively $0.474)$ in expectation. These coefficients are not statistically different from the $\rho_{\mathrm{WDQ}}(918)=0.30$ reported in WDQ $\left(H_{0}: \rho_{S M P}^{\prime}(35)=\rho_{W D Q}(918), p=0.761\right.$, respectively $p=0.253$, two-sided t-test $)$.

Moreover, the results in Table 1 indicate that any of the other bivariate correlation reported in WDQ would be substantially larger in the absence of censoring. For a more detailed description of the Monte Carlo study, see Appendix A of the supplemental material. 
Table 1: Monte Carlo study depicting the degree of downward bias in Pearson correlation coefficients with censored data

\begin{tabular}{|c|c|c|c|c|c|c|}
\hline \multirow[b]{3}{*}{ Censoring } & \multicolumn{6}{|c|}{$\begin{array}{l}\text { Pearson Correlation Coefficients under Censoring } \\
\text { Simulation results for } 10,000 \text { replications }\end{array}$} \\
\hline & \multicolumn{3}{|c|}{$\rho=0.42$} & \multicolumn{3}{|c|}{$\rho=0.57$} \\
\hline & mean & $\mathrm{Cl}, \mathrm{N}=918$ & $\mathrm{Cl}, \mathrm{N}=35$ & mean & $\mathrm{Cl}, \mathrm{N}=918$ & $\mathrm{Cl}, \mathrm{N}=35$ \\
\hline no censoring & 0.420 & {$[0.367-0.473]$} & {$[0.135-0.693]$} & 0.570 & {$[0.526-0.613]$} & {$[0.332-0.795$} \\
\hline 30 percent & 0.391 & [0.337-0.445] & [0.101-0.672] & 0.531 & {$[0.486-0.576]$} & {$[0.283-0.768]$} \\
\hline 35 percent & 0.384 & [0.330-0.439] & {$[0.091-0.665]$} & 0.522 & {$[0.476-0.567]$} & {$[0.270-0.760]$} \\
\hline 40 percent & 0.377 & {$[0.322-0.431]$} & {$[0.084-0.660]$} & 0.511 & {$[0.465-0.558]$} & {$[0.259-0.753]$} \\
\hline 45 percent & 0.368 & [0.314-0.423] & {$[0.075-0.654]$} & 0.500 & [0.453-0.547] & {$[0.247-0.746]$} \\
\hline 50 percent & 0.359 & [0.304-0.414] & {$[0.063-0.645]$} & 0.488 & [0.441-0.535] & {$[0.229-0.734]$} \\
\hline 55 percent & 0.349 & [0.294-0.405] & {$[0.049-0.636]$} & 0.474 & {$[0.426-0.522]$} & {$[0.209-0.722]$} \\
\hline 60 percent & 0.338 & [0.282-0.394] & [0.037-0.628] & 0.459 & [0.410-0.507] & {$[0.194-0.712]$} \\
\hline 65 percent & 0.325 & [0.269-0.381] & [0.024-0.618] & 0.442 & [0.392-0.491] & {$[0.175-0.699]$} \\
\hline 70 percent & 0.311 & [0.254-0.367] & {$[0.006-0.603]$} & 0.422 & {$[0.372-0.472]$} & {$[0.149-0.680]$} \\
\hline
\end{tabular}

Notes: The above results are based on data that mimic the correlation for achievement measures reported in SMP ( $\rho=0.42$ for SAT Verbal and $\rho=0.57$ for SAT Quantitative) in a sample of the size reported in WDQ and SMP respectively and with censored waiting times. In the Monte Carlo experiments (based on 10,000 Monte Carlo replications), we draw two correlated random variables from a standard normal distribution (waiting times and SAT verbal/quantitative scores) and censor the waiting time variable. Waiting times in WDQ are censored for $55 \%$ of the children ( $45 \%$ non-degreed mothers, $68 \%$ degreed mothers).

As SMP, WDQ also analyze associations between delay gratification and behavioral problems. The results are difficult to compare for two reasons. First, the constructs used to measure behavioral problems in both studies differ more widely than the achievement measures. Regarding academic achievement, the most important difference is a high-stakes test (parent-reported SAT) in SMP versus a low-stakes academic achievement measure (subtests of Woodcock-Johnson Psycho-Educational Battery Revised) in WDQ. For behavioral problems, however, SMP rely on measures that, by construction, are very closely linked to self-control and/or delay of gratification: (i) the Adolescent Coping Questionnaire contains many questions about self-control and delay of gratification (those were also most related) and when using the (ii) the California Child Q-Set personality inventory, the authors deliberately extracted those 11 sub-items from the 100 item that were deemed highly related to delay of gratification (one item even reads "Is unable to delay gratifications, cannot wait for satisfactions"). WDQ's main analysis, by contrast, draws on a behavioral composite score of externalizing and internalizing behaviors that comprises all 100 items 
(rated on a 3-point scale) from the Child Behavior Checklist. Second, WDQ, SMP, and Mischel, Shoda and Peake (1988) all report very mixed results as regards the relation of delay gratification with different measures of behavioral problems. Therefore, the absence of a significant relation between waiting time and externalizing and internalizing behavior reported in WDQ does not necessarily contradict SMP.

\section{Adding controls for family background and early cognitive ability}

In addition to reporting bivariate correlations, WDQ also study how the relation changes if covariates are added as control variables in a multivariate regression framework. We appreciate the authors' idea to explore the potential effect of interventions targeted at delay of gratification after the age of 36 or 54 months. However, we disagree that the analysis necessarily helps "to assess how much bias might be contained in longitudinal bivariate correlations between gratification delay and later outcomes as a result of failure to control for characteristics of children and their environments" (WDQ, p. 7). Instead, WDQ's approach to control for ability in all multivariate models can lead to an underestimation of the effect of interest whenever the measures of ability themselves are a function of (early) delay of gratification (see Angrist and Pischke, 2009, chapter 3.2.3 and our explanations below). This may be the case, e.g., if both characteristics develop jointly, or if the measurement of cognitive ability is affected by delay of gratification. A reanalysis of WDQ's data reveals that conditioning only on predetermined child background variables produces estimates which are more similar to the ones reported in the original studies.

\section{METHODS AND RESULTS II}

To illustrate the above argument, consider the following model:

$$
y_{i}=\beta_{0}+\beta_{D G} D G_{i}+X_{i}^{\prime} \beta+\varepsilon_{i},
$$

where $y_{i}$ is a later achievement measure, $D G_{i}$ is the delay of gratification measure and $X_{i}$ is a vector of control variables. Assume $A_{i}$ is an cognitive ability measure which depends on $D G_{i}$ as follows:

$$
A_{i}=\alpha_{i}+\alpha_{D G} D G_{i}
$$


If we estimated equation (1) and included $A_{i}$ as a control variable (as WDQ do, e.g., in Table 4), the overall effect of $D G_{i}$ on $y_{i}$ would be $\frac{d y_{i}}{d D G_{i}}=\beta_{D G}+\beta_{A} \alpha_{D G}$. Thus, given that $\beta_{A}$ and $\alpha_{D G}$ tend to be positive, $\beta_{D G}$ likely underestimates the true overall effect. There are several reasons for a potential dependence of $A_{i}$ on $D G_{i}$. Most importantly, if cognitive ability and delay of gratification develop jointly and interactively in children (see e.g. Cunha \& Heckman, 2007), equation (2) holds and a ceteris paribus interpretation of reduced form effects in observational data is difficult. In addition, even if $A_{i}$ develops independently of $D G_{i}$, the measure of cognitive ability might nevertheless be affected by noncognitive traits such as delay of gratification (see, e.g., Carlson \& Moses, 2001; Duckworth \& Seligman, 2006; Borghans et al., 2008). Besides, note that even if cognitive ability is measured earlier than delay of gratification, equation (2) may hold if delay of gratification is reasonably correlated over time.

To determine the effect of delay of gratification on later achievement in multivariate models with predetermined control variables only, we conduct a new analysis. Our empirical approach follows WDQ, but we only control for exogenous and predetermined variables such as sex, race, socio-economic status and parental ability, i.e., excluding any variables that tend to proxy early (cognitive) ability in children, except those that were administered pre-birth. We preregistered the analysis before we had access to the data (see Falk, Kosse and Pinger (2018) for details). Under a matching on observables assumption this analysis unveils the effect of delay of gratification on later achievement holding fixed characteristics that were determined at birth. As displayed in Panel A of Table 2, the coefficients in the conditional model reduce by much less when compared to WDQ's estimates (one third instead of two thirds) and remain highly significant $(p<0.001)$. Moreover, if we standardize all variables in a way that allows for a direct comparison of the estimates to the correlation coefficients reported in SMP, effect sizes increase even further (Table 2, Panel B). 
Table 2: Associations Between Delay of Gratification at Age 54 Months and Later Measures of Academic Achievement for Children of Mothers Without College Degrees

Achievement composite

Grade 1

Age 15

(1)

(2)

(3)

(4)

Panel A: Variables standardized among all children as in WDQ (mothers with \& without college degree)

Delay minutes

$0.279 * \quad 0.181^{*}$

$0.236^{*}$

$0.137^{*}$

(0.038)

(0.036)

(0.037)

Panel B: Variables standardized within sample (only mothers without college degree)
Delay minutes
$0.305^{*}$
$0.198^{*}$
$0.286^{*}$
$0.166^{*}$
(0.041)
(0.040)
(0.045)

Controls: Predetermined background characteristics

No Yes

No

Yes

Note: $n=552$. For details on the estimations see WDQ. The table shows standardized coefficients (with standard errors in parentheses). Columns 1 and 3 show estimates for models that only contain delay of gratification as a covariate. In Panel B the variables are standardized within sample, i.e., among children of mothers without college degree. Consequently, the coefficients in Columns 1 and 3 in Panel B are equivalent to Pearson correlation coefficients and can therefore be compared to the Pearson correlation coefficients reported in SMP. Columns 2 and 4 report estimates for models that control for the following predetermined background characteristics: sex of child, race dummies, log of family income, mother's age at birth (years), mother's education (years) and mother's PPVT score * $p<.05$.

\section{Discussion and conclusion}

We strongly appreciate WDQ's contribution and initiative to replicate SMP's analysis in a larger and more diverse sample. However, given the fundamental differences across studies and in light of the active academic and public debate about child inequality, early ability measures and intervention possibilities, we propound a more cautious interpretation. Due to fundamental differences in measures, downwardbiased estimates and difficulties in interpreting effect sizes in empirical models with other ability measures as control variables, the new findings should not be interpreted as a falsification of the original marshmallow studies. Instead, our reanalysis of the data reveals that conditioning only on predetermined variables yields estimates which are much closer to the ones reported in the original studies.

WDQ provide useful suggestive evidence that the early environment shapes a child's ability to delay gratification (see the correlations in Table 7 in WDQ), which is in accordance with a body of related evidence (e.g. Kosse \& Falk, 2016; Deckers, Falk, Kosse, Pinger, \& Schildberg-Hörisch, 2017). Nonetheless, a detailed understanding of the dynamic process describing how parental background factors and investments form children's delay of gratification, cognitive achievement and outcomes requires more 
refined structural models that allow for simultaneity, self- and cross-productivities (Cunha \& Heckman, 2007). In addition, we agree with WDQ that targeted intervention studies are needed to provide truly exogenous variation in investments and thus in delay of gratification. Arguably, such interventions would take most effect if they did not teach "waiting strategies", but instead provided an enhanced social environment to children who are deprived by the "accident of birth" (Heckman 2008, 2013). 


\section{References}

Angrist, J. D., \& Pischke, J.-S. (2008). Mostly harmless econometrics: An empiricist's companion. Princeton University Press.

Borghans, L., Meijers, H., \& Ter Weel, B. (2008). The role of noncognitive skills in explaining cognitive test scores. Economic Inquiry, 46(1), 2-12.

Carlson, S. M., \& Moses, L. J. (2001). Individual differences in inhibitory control and children's theory of mind. Child Development, 72(4), 1032-1053.

Cunha, F., \& Heckman, J. J. (2007). The technology of skill formation. American Economic Review, 97(2), 31-47.

Duckworth, A. L., \& Seligman, M. E. (2006). Self-discipline gives girls the edge: Gender in self-discipline, grades, and achievement test scores. Journal of Educational Psychology, 98(1), 198.

Deckers, T., Falk, A., Kosse, F., Pinger, P., \& Schildberg-Hörisch, H. (2017). Socio-economic status and inequalities in children's IQ and economic preferences. HCEO Working Paper Series, 2017-088.

Falk, A., \& Kosse, F. (2016). Breastfeeding duration, early life circumstances and the formation of human preferences. HCEO Working Paper Series, 2016-036.

Falk, A., Kosse, F., \& Pinger, P. (2018, October 12). Preregistration of analysis: Revisiting the Marshmallow Test: On the Interpretation of Replication Results. https://doi.org/10.17605/OSF.IO/5JPT4 Heckman, J. J. (2008). Schools, skills, and synapses. Economic Inquiry, 46(3), 289-324.

Heckman, J. J. (2013). Giving kids a fair chance. MIT Press.

Mischel, W., Ebbesen, E. B., \& Raskoff Zeiss, A. (1972). Cognitive and attentional mechanisms in delay of gratification. Journal of Personality and Social Psychology, 21(2), 204-218.

Mischel, W., Shoda, Y., \& Peake, P. K. (1988). The nature of adolescent competencies predicted by preschool delay of gratification. Journal of Personality and Social Psychology, 54(4), 687.

Mischel, W., Shoda, Y., \& Rodriguez, M. I. (1989). Delay of gratification in children. Science, 244(4907), 933-938.

Shoda, Y., Mischel, W., \& Peake, P. K. (1990). Predicting adolescent cognitive and self-regulatory competencies from preschool delay of gratification: Identifying diagnostic conditions. Developmental Psychology, 26(6), 978-986. 
Watts, T. W., Duncan, G. J., \& Quan, H. (2018). Revisiting the Marshmallow Test: A Conceptual Replication Investigating Links Between Early Delay of Gratification and Later Outcomes. Psychological Science, 29(7), $1-12$. 